\title{
Joint Estimation of Carrier-Frequency and Sampling-Frequency Offsets for SC-FDE Systems on Multipath Fading Channels
}

\author{
Ping-Hung Chiang, Student Member, IEEE, Ding-Bing Lin, Member, IEEE, Hsueh-Jyh Li, Senior Member, IEEE, \\ and Gordon L. Stüber, Fellow, IEEE
}

\begin{abstract}
With single-carrier frequency-domain equalization, the carrier-frequency and sampling-frequency offsets are embedded in the phases of complex frequency-domain signal components. This paper proposes a sub-block processing to extract the phases and applies the least-squares regression to jointly estimate the offsets. The effectiveness of the proposed SC-FDE receiver is demonstrated on multipath fading channels.
\end{abstract}

Index Terms-Carrier frequency offset, least-squares estimation, sampling frequency offset, single-carrier frequency-domain equalization (SC-FDE), synchronization.

\section{INTRODUCTION}

$\mathbf{S}$ INGLE-CARRIER frequency-domain equalization (SCFDE) [1], [2] and orthogonal frequency division multiplexing (OFDM) [3] are closely related; both are effective methods for combating inter-symbol interference (ISI) and have been adopted as the air interfaces of IEEE802.16 wireless metropolitan area network (WirelessMAN) standard [4].

This paper focuses on fine synchronization of an SCFDE receiver. The residual carrier-frequency offset (CFO) and sampling-frequency offset (SFO) cause the inter-carrier interference (ICI) and the latter also causes the fast Fourier transform (FFT) window to drift; therefore, these two unknowns need to be estimated and compensated for. For an SCFDE receiver, the CFO and SFO are embedded in the phases of complex frequency-domain signal components. Thus their joint estimation can be performed in the frequency domain, provided that the frequency-domain signal components are known. However, the frequency-domain signal components are the mixture of the data and pilot symbols and, hence, are unknown to the receiver. Two direct solutions to this problem are the frequency-domain pilot method [5] and the decisiondirected scheme [6]. Unfortunately, the former increases the transmitter complexity and results in pilot sequences having a high peak-to-average power ratio (PAPR), while the latter increases the receiver latency and complexity and suffers from error propagation.

We propose a novel sub-block processing to solve the problem, which preserves the benefits of single-carrier transmission

Paper approved by E. Erkip, the Editor for Cooperation Diversity of the IEEE Communications Society. Manuscript received August 7, 2006; revised April 3, 2007 and September 12, 2007.

P.-H. Chiang and G. Stüber are with the School of Electrical and Computer Engineering, Georgia Tech, Atlanta, GA 30332 USA (e-mail: pinghungchiang@gmail.com; stuber@ece.gatech.edu).

P.-H. Chiang and H.-J. Li are with the Graduate Institute of Communication Engineering, National Taiwan University, Taipei, Taiwan, R.O.C. (e-mail: hili@ew.ee.ntu.edu.tw)

D.-B. Lin is with Department of Electronic Engineering, National Taipei University of Technology, Taipei, Taiwan, R.O.C. (e-mail: dblin@en.ntut.edu.tw).

Digital Object Identifier 10.1109/TCOMM.2008.060456. and has a lower complexity than alternative approaches. With the sub-block structure, the frequency-domain signal components are known at the pilot sub-blocks and, hence, the phases can be extracted and used in the least-squares (LS) regression to jointly estimate the CFO and SFO. Here, the linear leastsquares (LLS) [7] and simple weighted least-squares (SWLS) [8] estimators are applied, and the latter is shown to be robust to channel frequency-selectivity. For the additive white Gaussian noise (AWGN) channel, the LLS estimator is proven to be unbiased and its theoretical mean-squared estimation error (MSE) is derived. Finally, the performances of the estimators on quasi-static and time-varying multipath fading channels are evaluated.

\section{SySTEM MOdEL}

\section{A. Signal Model with Synchronization Errors}

SC-FDE uses a cyclic prefixed block-transmission technique [1], [2]. As shown in Fig. 1(a), each of the transmitted blocks consists of $N+N_{G}$ channel symbols. For each block, the cyclic prefix (CP), which is a copy of the last $N_{G}$ symbols, acts as a guard to avoid inter-block-interference (IBI). The pilot word, which is a sequence of $N_{P}$ pilot symbols, is used for synchronization and channel estimation. Let $x(t)$ be the complex baseband transmitted signal and $T_{S}$ be the sampling period. Then the $n$th time-domain sample in the $i$ th block is expressed as $x\left(\left[i\left(N+N_{G}\right)+N_{G}+n\right] T_{S}\right)=$ $x_{n, i}$, for $-N_{G} \leq n \leq N-1$. In this paper, both the data and pilot symbols are assumed to be of energy $E_{S}$. Also, the $N$-point discrete-Fourier transform (DFT) pair is defined as: $x_{n, i}=\frac{1}{N} \sum_{k=-N / 2+1}^{N / 2} X_{k, i} e^{j 2 \pi n k / N}$ and $X_{k, i}=$ $\sum_{n=0}^{N-1} x_{n, i} e^{-j 2 \pi n k / N}$, where $X_{k, i}$ is the $k$ th subcarrier symbol.

In the presence of CFO $\varepsilon / N T_{S}$ and SFO $\delta T_{S}$, we define $n_{i} \equiv i\left(N+N_{G}\right)+N_{G}+n$ and write the $n$th received sample in the $i$ th block as (1), which is shown at the next page, where $H_{k, i}$ is the channel frequency response of the $k$ th subcarrier and $v_{n, i} \sim \mathcal{C N}\left(0, N_{0}\right)$ is the time-domain noise. By defining $\lambda_{N}(\varphi)=\sin (\pi \varphi) /[N \sin (\pi \varphi / N)]$ and $\varphi_{q k} \equiv(1+\delta)(\varepsilon+q)-k$, the $N$-point DFT of (1) is derived as (2), which is shown at the next page, where $C_{k, i}$ and $V_{k, i}$ are the ICI and frequency-domain noise, respectively. In light of (2), for each subcarrier symbol $X_{k, i}$, the CFO and SFO cause the magnitude attenuation, phase rotation, and ICI. Thereupon, the CFO and SFO should be estimated and compensated for.

\section{B. Basic Concepts of Estimation}

To remove the phase of the channel frequency response, we assume $\varepsilon$ and $\delta$ being small and $H_{k, i} \approx H_{k, i-1}$ and write the 


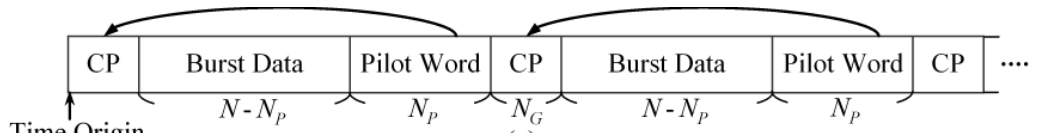

(a)

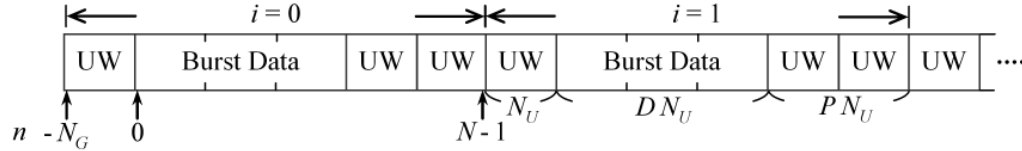

(b)

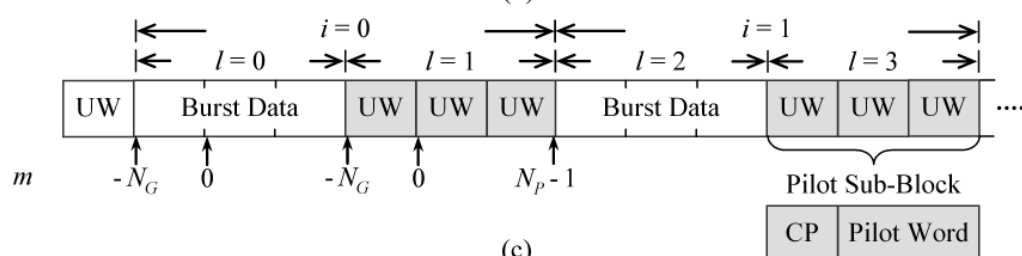

(c)

Fig. 1. Block structures of the (a) general SC-FDE system, (b) WirelessMAN-SCa PHY, and (c) sub-block processing, for $B=2, D=3$, and $P=2$.

$$
\begin{gathered}
z_{n, i}=e^{j 2 \pi n_{i}(1+\delta) \varepsilon / N} \frac{1}{N} \sum_{k=-N / 2+1}^{N / 2} e^{j 2 \pi n k / N} e^{j 2 \pi n_{i} \delta k / N} H_{k, i} X_{k, i}+v_{n, i}, 0 \leq n \leq N-1, \\
Z_{k, i}=\lambda_{N}\left(\varphi_{k k}\right) e^{j \pi\left[2 i\left(N+N_{G}\right)+2 N_{G}+N-1\right] \varphi_{k k} / N} H_{k, i} X_{k, i}+C_{k, i}+V_{k, i}, \quad-N / 2+1 \leq k \leq N / 2, \\
\underline{Z}_{k, l}=\lambda_{N_{P}}\left(\underline{\varphi}_{k k}\right) e^{j \pi\left[2 l\left(N_{P}+N_{G}\right)+4 N_{G}+N_{P}-1\right] \underline{\varphi}_{k k} / N_{P}} \underline{H}_{k, i} \underline{A}_{k}+\underline{C}_{k, l}+\underline{V}_{k, l}, \quad-N_{P} / 2+1 \leq k \leq N_{P} / 2,
\end{gathered}
$$

conjugate product of two consecutive blocks as

$$
\begin{aligned}
Y_{k, i} & =Z_{k, i} Z_{k, i-1}^{*} \\
& \approx e^{j 2 \pi\left(N+N_{G}\right) \varphi_{k k} / N}\left|H_{k, i}\right|^{2} X_{k, i} X_{k, i-1}^{*}+\text { noise.(3) }
\end{aligned}
$$

If $X_{k, i}$ and $X_{k, i-1}$ are known, the phase $\varphi_{k k}$ can be obtained by taking the argument of $Y_{k, i} / X_{k, i} X_{k, i-1}^{*}$, and $\varepsilon$ and $\delta$ can be extracted by applying the linear regression [7] to a straight line $\varphi_{k k} \approx \varepsilon+\delta k$. However, $X_{k, i}$ and $X_{k, i-1}$ are mixtures of the time-domain data and pilot symbols and, hence, are unknown. To solve this difficulty, the sub-block processing is introduced.

\section{Sub-Block Processing}

The block structure of the WirelessMAN-SCa PHY [4] is depicted in Fig. 1(b), where the unique word (UW) possesses the constant amplitude zero auto-correlation (CAZAC) property. The CP and pilot word, respectively, consist of one and $P$ UWs. To simplify the system design, the length of the burst data is an integer multiple of the length of a UW, i.e., $N-N_{P}=D N_{U}$.

As shown in Fig. 1(c), for sub-block processing, the block boundaries are shifted right by $N_{G}$ samples, and each block of length $N+N_{G}$ is partitioned into $B$ sub-blocks, each of length $N_{P}+N_{G}$. More specifically, the $i$ th block is partitioned into $B$ sub-blocks with sub-block indices $l=i B+0, i B+$ $1, \cdots, i B+(B+1)$, for $i \geq 0$. In each pilot sub-block, i.e., the last sub-block of each block, there are $P+1 \mathrm{UWs}$; the first UW acts as the CP, while the last $P$ UWs act as the pilot word. Since the pilot word $\left\{\underline{a}_{m}, 0 \leq m \leq N_{P}-1\right\}$ consists of $P$ identical UWs possessing the CAZAC property, the amplitudes of pilot symbols are $\left|\underline{a}_{m}\right|=\sqrt{E_{S}}$ for all $m$, and the squared amplitudes of pilot subcarrier symbols are

$$
\left|\underline{A}_{k}\right|^{2}= \begin{cases}P^{2} N_{U} E_{S}, & \text { if } k \in \mathcal{A}, \\ 0, & \text { else, }\end{cases}
$$

where $\underline{A}_{k}$ is the $N_{P}$-point DFT of $\underline{a}_{m}$ and $\mathcal{A}=\{k: k=$ $q P$ and $\left.-N_{U} / 2+1 \leq q \leq N_{U} / 2\right\}$.

Subsequently, we analyze the effects of the CFO and SFO for the pilot sub-blocks with indices $l \in \mathcal{L}$, where $\mathcal{L}=\{l: l=$ $i B+(B-1)$, for $i \geq 0\}$. First, the $m$ th time-domain sample in the $l$ th sub-block is $x\left(\left[l\left(N_{P}+N_{G}\right)+2 N_{G}+m\right] T_{S}\right)=\underline{x}_{m, l}$, for $-N_{G} \leq m \leq N_{P}-1$ and $l \in \mathcal{L}$, where $\underline{x}_{m, l}=\underline{a}_{m}$, for $0 \leq m \leq N_{P}-1$, and $\underline{x}_{m, l}=\underline{a}_{m+N_{P}}$, for $-N_{G} \leq m \leq$ -1 . Considering the effects of the CFO and SFO, defining $\underline{\varphi}_{q k} \equiv(1+\delta)(\underline{\varepsilon}+q)-k$ and $\underline{\varepsilon} \equiv \varepsilon N_{P} / N$, following the derivations given in (1) and (2), and using the $N_{P}$-point DFT, one can derive the frequency-domain samples of a pilot subblock as (5), which is shown below Fig. 1, where $\underline{H}_{k, i}$ is the channel frequency response, $\underline{C}_{k, l}$ is the ICI, and $\underline{V}_{k, l} \sim$ $\mathcal{C N}\left(0, N_{P} N_{0}\right)$ is the noise. By neglecting the ICI for small $\varepsilon$ and $\delta$, one can write the conjugate product of two consecutive pilot sub-blocks as

$$
\begin{aligned}
\underline{Y}_{k, i} & =\underline{Z}_{k, i B+(B-1) \underline{Z}_{k,(i-1) B+(B-1)}^{*}} \\
& \approx e^{j 2 \pi\left(N+N_{G}\right) \underline{\varphi}_{k k} / N_{P}}\left|\underline{H}_{k, i}\right|^{2}\left|\underline{A}_{k}\right|^{2}+\text { noise },
\end{aligned}
$$

provided that $\underline{H}_{k, i} \approx \underline{H}_{k, i-1}$. Then the total frequency offsets $\underline{\varphi}_{k k}$ can be estimated on the non-zero pilot subcarriers, i.e.,

$$
\underline{\hat{\varphi}}_{k k, i}=\frac{N_{P}}{2 \pi\left(N+N_{G}\right)} \arg \left(\underline{Y}_{k, i}\right), \text { for } k \in \mathcal{A},
$$

where $\arg (\cdot)$ denotes the argument of a complex number. 


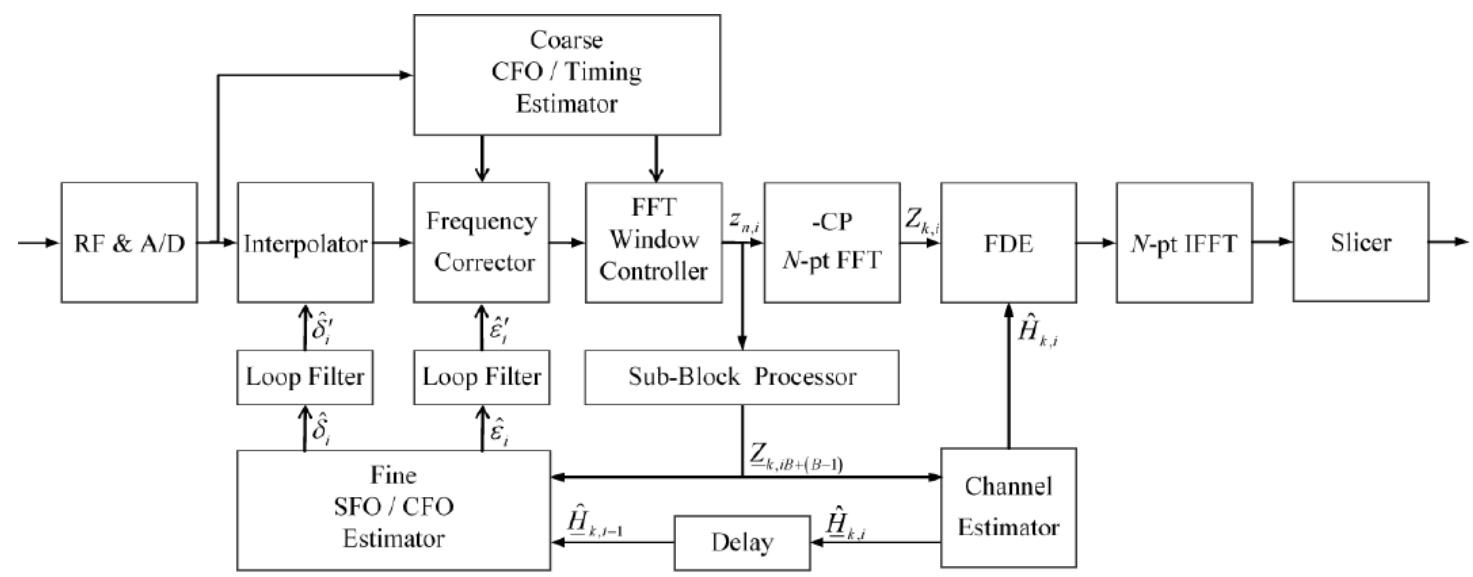

Fig. 2. The proposed receiver architecture.

\section{JOINT ESTIMATION OF CFO AND SFO}

For small $\varepsilon$ and $\delta$, the product term $\varepsilon \delta$ in $\underline{\varphi}_{k k}$ is neglected. Thus the joint one-shot estimation of the $\mathrm{CFO}$ and SFO can be achieved by employing $\underline{\varphi}_{k k}=\underline{\varepsilon}+\delta k$ and performing LS regression on the estimates of the total frequency offsets given in (7). To avoid the effects of the direct-current (DC) offset and phase non-linearity, the subcarriers at DC and the band edges are not used in the regression and only the non-zero pilot subcarriers with indices $k \in \mathcal{K}$ are employed, where $\mathcal{K}=\{k: k= \pm P, \pm 2 P, \cdots, \pm Q P\}$. From our experience, a rule of thumb is to choose $Q$ as $Q=\left\lfloor 0.4 N_{U}\right\rfloor$, where $\lfloor\cdot\rfloor$ denotes the floor function.

The SWLS estimator of the CFO and SFO collects $\underline{\hat{\varphi}}_{k k, i}$ from the non-zero pilot subcarriers, assigns each pilot subcarrier a different weight $w_{k, i}$, and performs LS regression via [8]

$$
\begin{gathered}
\hat{\varepsilon}_{i}=\frac{N}{N_{P}} \cdot \frac{\sum_{k \in \mathcal{K}} w_{k, i} \underline{\hat{\varphi}}_{k k, i}}{\sum_{k \in \mathcal{K}} w_{k, i}}, \\
\hat{\delta}_{i}=\frac{\sum_{k \in \mathcal{K}} k w_{k, i} \underline{\hat{\varphi}}_{k k, i}}{\sum_{k \in \mathcal{K}} k^{2} w_{k, i}},
\end{gathered}
$$

where $w_{k, i}=\left|\underline{H}_{k, i}\right|^{2}$ and is used to compensate for different levels of fading encountered at different subcarriers. During each block, the channel frequency response is, in general, unknown before synchronization takes place. However, we can simply use the channel estimate of the last block in the synchronization for the present block, i.e., $w_{k, i}=\left|\underline{\hat{H}}_{k, i-1}\right|^{2}$ and $w_{k, 0}=1$, where $\underline{\hat{H}}_{k, i}$ can be obtained by using a pilotaided channel estimation based on the sub-block structure [10]. Noteworthily, the SWLS estimator reduces to the LLS estimator, provided that equal weights (i.e. $w_{k, i}=1$ ) are utilized.

As depicted in Fig. 2, to smooth the fluctuation of one-shot estimates $\hat{\varepsilon}_{i}$ and $\hat{\delta}_{i}$, we incorporate a closed-loop tracking [9] into the proposed receiver architecture, i.e.,

$$
\begin{aligned}
& \hat{\varepsilon}_{i}^{\prime}=\hat{\varepsilon}_{i-1}^{\prime}+\gamma_{\varepsilon} \hat{\varepsilon}_{i}, \quad \hat{\varepsilon}_{0}^{\prime}=0, \\
& \hat{\delta}_{i}^{\prime}=\hat{\delta}_{i-1}^{\prime}+\gamma_{\delta} \hat{\delta}_{i}, \quad \hat{\delta}_{0}^{\prime}=0,
\end{aligned}
$$

where $\gamma_{\varepsilon}$ and $\gamma_{\delta}$ are step sizes taking on the values between $(0,1)$. With the closed-loop tracking, the magnitudes of the residual CFO and SFO decrease and converge to small values.

\section{Performance Analysis}

In this section, assuming small $\varepsilon$ and $\delta$, we prove that the LLS estimator is unbiased and derive its MSE. For the AWGN channel (i.e. $\underline{H}_{k, i}=1$ ), substituting (4) into (6) and ignoring the product of two noise terms (for high signal-to-noise ratio (SNR) region), one can rewrite (6) as

$\underline{Y}_{k, i}=e^{j 2 \pi\left(N+N_{G}\right) \underline{\varphi}_{k k} / N_{P}} P^{2} N_{U} E_{S}+\underline{W}_{k, i}$, for $k \in \mathcal{K}$,

where $\underline{W}_{k, i}$ is the noise distributed as $\mathcal{C N}\left(0,2 P^{3} N_{U}^{2} E_{S} N_{0}\right)$. We define $\underline{\tilde{Y}}_{k, i} \equiv \underline{Y}_{k, i} / P^{2} N_{U} E_{S}$ and $\alpha \equiv \exp [-j 2 \pi(N+$ $\left.\left.N_{G}\right) \underline{\varepsilon} / N_{P}\right]$, and write their product as

$$
\alpha \underline{\tilde{Y}}_{k, i}=e^{j 2 \pi\left(N+N_{G}\right) \delta k / N_{P}}+\alpha \underline{\tilde{W}}_{k, i},
$$

where $\underline{\tilde{W}}_{k, i} \sim \mathcal{C N}\left(0,2 N_{0} / P E_{S}\right)$. By substituting (7) into (8) and using $\arg \left(\underline{\tilde{Y}}_{k, i}\right)=\arg \left(\underline{Y}_{k, i}\right)$, the estimate is rewritten as

$$
\hat{\varepsilon}_{i}=\frac{N}{4 \pi Q\left(N+N_{G}\right)} \sum_{k \in \mathcal{K}} \arg \left(\underline{\tilde{Y}}_{k, i}\right) .
$$

Then, in light of (13) and (14), the estimation error of the CFO can be derived as

$$
\begin{aligned}
e_{\varepsilon, i} & \equiv \hat{\varepsilon}_{i}-\varepsilon \\
& =\frac{N}{4 \pi Q\left(N+N_{G}\right)} \sum_{k \in \mathcal{K}} \arg \left(\alpha \underline{\tilde{Y}}_{k, i}\right) \\
& \approx \frac{N}{4 \pi Q\left(N+N_{G}\right)} \sum_{k \in \mathcal{K}} \Im\left(\alpha \underline{\tilde{Y}}_{k, i}\right) \\
& \approx \frac{N}{4 \pi Q\left(N+N_{G}\right)} \sum_{k \in \mathcal{K}} \Im\left(\alpha \underline{\tilde{W}}_{k, i}\right),
\end{aligned}
$$

where $\Im(\cdot)$ denotes the imaginary part of a complex number, the third line is due to the approximation of $\theta \approx \sin \theta$ for small $\theta$, and the last line is due to the assumption of small $\delta$. Using (15) and recognizing that $\Im\left(\alpha \underline{\tilde{W}}_{k, i}\right) \sim \mathcal{N}\left(0, N_{0} / P E_{S}\right)$, it can be shown that $\hat{\varepsilon}_{i}$ is unbiased, i.e., $\mathrm{E}\left[e_{\varepsilon, i}\right]=0$, and its MSE is 

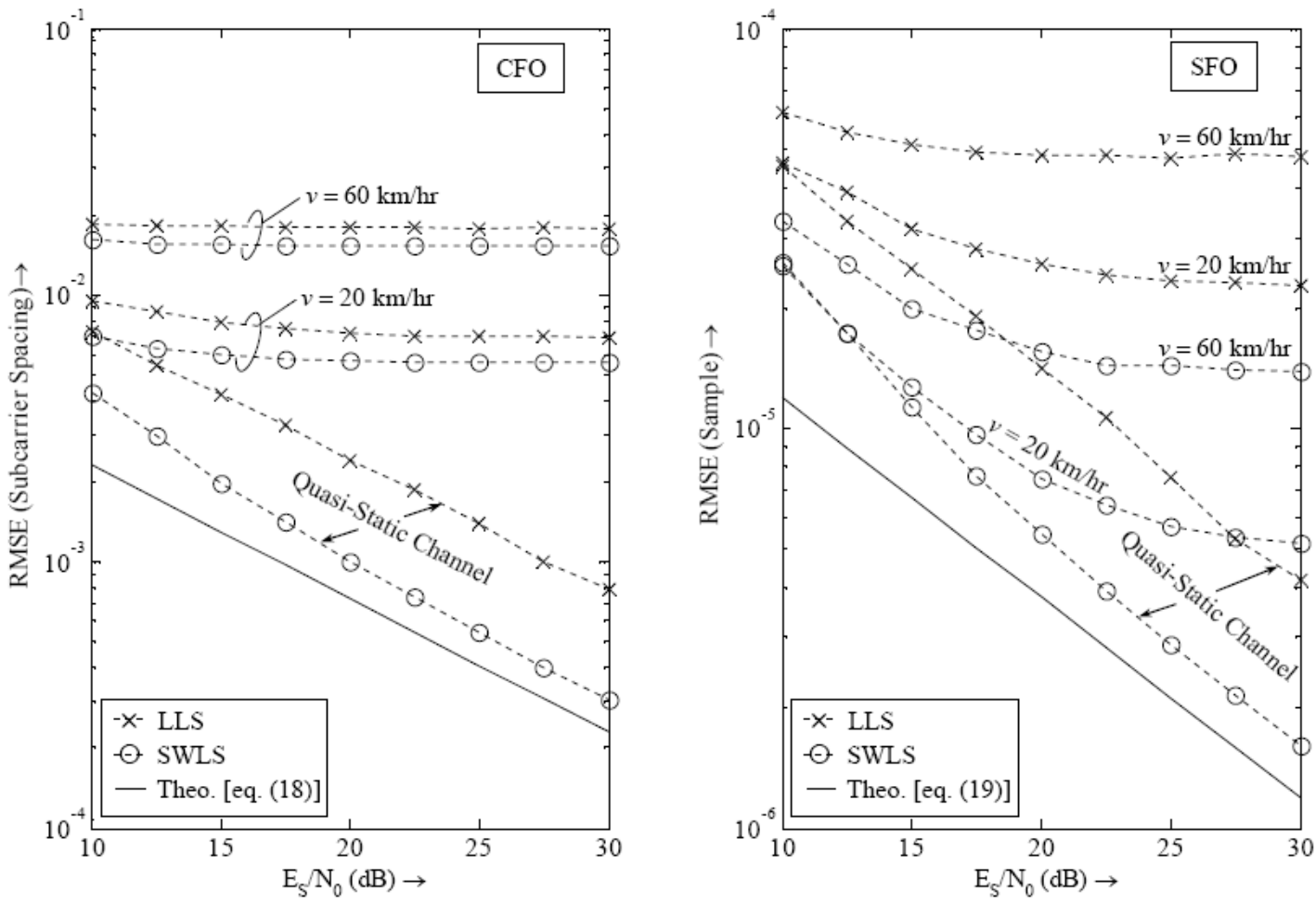

Fig. 3. RMSEs of the closed-loop tracking in multipath fading channels for $\varepsilon=0.05$ and $\delta=-20 \mathrm{ppm}$.

derived as

$$
\begin{aligned}
\operatorname{MSE}\left(\hat{\varepsilon}_{i}\right) & \equiv \mathrm{E}\left[e_{\varepsilon, i}^{2}\right] \\
& =\frac{N^{2}}{8 \pi^{2} P Q\left(N+N_{G}\right)^{2} \cdot E_{S} / N_{0}} .
\end{aligned}
$$

Similarly, one can show that $\hat{\delta}_{i}$ is unbiased and its MSE is

$$
\begin{aligned}
\operatorname{MSE}\left(\hat{\delta}_{i}\right) & \equiv \mathrm{E}\left[e_{\delta, i}^{2}\right] \\
& =\frac{N_{U}^{2}}{4 \pi^{2} P\left(2 Q^{3}+3 Q^{2}+Q\right)\left(N+N_{G}\right)^{2} \cdot E_{S} / N_{0}} .
\end{aligned}
$$

Moreover, by assuming that the tracking error is small enough to allow for loop linearization [9, Ch. 3], the MSEs of $\hat{\varepsilon}_{i}^{\prime}$ and $\hat{\delta}_{i}^{\prime}$ in the steady state are derived as:

$$
\begin{aligned}
& \operatorname{MSE}\left(\hat{\varepsilon}_{i}^{\prime}\right)=\frac{\gamma_{\varepsilon}^{2}}{2-\gamma_{\varepsilon}} \operatorname{MSE}\left(\hat{\varepsilon}_{i}\right), \\
& \operatorname{MSE}\left(\hat{\delta}_{i}^{\prime}\right)=\frac{\gamma_{\delta}^{2}}{2-\gamma_{\delta}} \operatorname{MSE}\left(\hat{\delta}_{i}\right) .
\end{aligned}
$$

\section{Numerical Results}

The performance of the proposed receiver depicted in Fig. 2 has been evaluated with the system parameters summarized in Tab. I and the SUI-4 channel model specified in [11].

Fig. 3 demonstrates the root-mean-squared errors (RMSEs) of the LLS and SWLS estimators with the closed-loop tracking in quasi-static and time-varying multipath Rayleigh fading channels, where the CFO and SFO are $\varepsilon=0.05$ subcarrier spacings and $\delta=-20 \mathrm{ppm}$, respectively. The RMSEs of the LLS estimator with the closed-loop tracking in the AWGN channel are also provided as the benchmarks. Due to the
TABLE I

SYSTEM PARAMETERS

\begin{tabular}{ccc}
\hline \hline Carrier Frequency & $f_{C}$ & $5 \mathrm{GHz}$ \\
\hline System Bandwidth & $1 / T_{S}$ & $3.5 \mathrm{MHZ}$ \\
\hline Number of Subcarriers & $N$ & 512 \\
\hline Length of CP & $N_{G}$ & 16 \\
\hline Length of Pilot Word & $N_{P}$ & 32 \\
\hline Length of UW & $N_{U}$ & 16 \\
\hline $\begin{array}{c}\text { Number of Subcarriers } \\
\text { Used in Regression }\end{array}$ & $2 Q$ & 12 \\
\hline Modulation & \multicolumn{2}{c}{ 16QAM } \\
\hline \hline
\end{tabular}

weighting based on the squared-magnitude of the subcarrier frequency response, the SWLS estimator is robust to the channel frequency-selectivity and is of a smaller RMSE than the LLS estimator in both quasi-static and time-varying channels. For the time-varying channels, due to the additional ICI caused by the channel variation within a block duration and the failure of the assumption, $\underline{H}_{k, i} \approx \underline{H}_{k, i-1}$ [cf. (6)], the performances of both LLS and SWLS estimators are degraded and the RMSE floors present.

\section{CONCLUSIONS}

Based on the sub-block processing, the frequency-domain joint estimation of the CFO and SFO for SC-FDE systems was proposed. Both the LLS and SWLS regressions were considered, where the latter was shown to be robust to the channel frequency-selectivity. For the AWGN channel, the LLS estimator was proven to be unbiased and the MSEs 
were derived. Finally, the proposed receiver was tested in both quasi-static and time-varying multipath fading channels and its superiority has been demonstrated in the numerical results.

\section{REFERENCES}

[1] H. Sari, G. Karam, and I. Jeanclaude, "Transmission techniques for digital terrestrial TV broadcasting," IEEE Commun. Mag., vol. 33, no. 2, pp. 100-109, Feb. 1995.

[2] D. Falconer, S. L. Ariyavisitakul, A. B. Seeyar, and B. Eidson, "Frequency domain equalization for single-carrier broadband wireless systems," IEEE Commun. Mag., vol. 40, no. 4, pp. 58-66, Apr. 2002.

[3] Y. Li and G. L. Stüber, Orthogonal Frequency Division Multiplexing for Wireless Communications. New York: Springer, 2006.

[4] Air Interface for Fixed Broadband Wireless Access Systems (IEEE Std 802.16-2004), June 2004.

[5] H. Yu, M. S. Kim, and J. Y. Ahn, "Carrier frequency and timing offset tracking scheme for SC-FDE systems," in Proc. IEEE Int. Symp. Personal, Indoor and Mobile Radio Communications, pp. 1-5, Sept. 2003.
[6] K. Shi, E. Serpedin, and P. Ciblat, "Decision-directed fine synchronization in OFDM systems," IEEE Trans. Commun., vol. 53, no. 3, pp. 408-412, Mar. 2005.

[7] I. H. Hwang, H. S. Lee, and K. W. Kang, "Frequency and timing period offset estimation technique for OFDM systems," IEE Electron. Lett., vol. 34, no. 6, pp. 520-521, Mar. 1998.

[8] P. Y. Tsai, H. Y. Kang, and T. D. Chiueh, "Joint weighted least-squares estimation of carrier-frequency offset and timing offset for OFDM systems over multipath fading channels," IEEE Trans. Veh. Technol., vol. 54, no. 1, pp. 211-223, Jan. 2005.

[9] U. Mengali and A. N. D'Andrea, Synchronization Techniques for Digital Receivers. New York: Plenum, 1997.

[10] P. H. Chiang, G. L. Stüber, D. B. Lin, and H. J. Li, "Pilot-aided fine synchronization for SC-FDE systems on multipath fading channels," in Proc. IEEE Int. Conf. on Communications, pp. 2853-2858, June 2007.

[11] Channel Models for Fixed Wireless Applications (IEEE 802.16a-03/01), June 2003. 